\title{
Event-Triggered Pinning Control of Complex Networks with Switching Topologies
}

\author{
Antonio Adaldo, Francesco Alderisio, Davide Liuzza, Guodong Shi, \\ Dimos V. Dimarogonas, Mario di Bernardo and Karl Henrik Johansson
}

\begin{abstract}
This paper investigates the problem of eventtriggered pinning control for the synchronization of networks of nonlinear dynamical agents onto a desired reference trajectory. The pinned agents are those that have access to the reference trajectory. We consider both static and switching topologies. We prove that the system is well posed and identify conditions under which the network achieves exponential convergence. A lower bound for the rate of convergence is also derived. Numerical examples demonstrating the effectiveness of the results are provided.
\end{abstract}

\section{INTRODUCTION}

Multi-agent systems have attracted a large amount of research in the past few decades - see for example [1] because they provide a suitable model for a remarkably wide spectrum of phenomena spanning the fields of biology, social sciences, physics, economics and engineering - see for example [2].

Pinning control is one of the possible inflections of the problem of multi-agent coordination. In the pinning control problem it is required that a set of interconnected dynamical systems synchronize onto a reference trajectory. A small fraction of the agents can be selected to receive a direct feedback from the reference. Such agents are called pins or pinned agents. The remaining agents are influenced only by their connections in the network. As opposed to more traditional consensus problems, the reference trajectory is supposed to be a solution of the uncoupled node dynamics, apriori known and corresponding to a control objective. When the dynamics of the agents and of the controllers are given, selection of the pins is left as the crucial element of the control design.

Research on pinning control usually focuses on finding sufficient conditions for convergence relating to the agents' dynamics and the connections' topology - see for example [3]-[8] - or on criteria for optimal selection of the agents to control - see for example [9], [10] - or on designing adaptive control laws - see for example [11], [12].

A. Adaldo, D. Liuzza, G. Shi, D.V. Dimarogonas and K.H. Johansson are with ACCESS Linnaeus Center and School of Electrical Engineering, Royal Institute of Technology, Stockholm, Sweden. Emails: \{adaldo, liuzza, guodongs, dimos, kallej\}@kth.se.

F. Alderisio and M. di Bernardo are with Department of Engineering Mathematics, University of Bristol, UK. Emails: $\{\mathrm{m}$. dibernardo, f.alderisio\}@bristol.ac.uk.

M. di Bernardo is also with Department of Electrical Engineering and Information Technology, University of Naples Federico II, Italy. Email: mario.dibernardo@unina.it

This work was partly supported by the Swedish Research Council and the Knut och Alice Wallenberg Foundation. The first two authors wish to thank the EU for providing funding to visit KTH under the Erasmus programme.
In many realistic scenarios a set of dynamical systems are required to synchronize over a network with a timevarying topology - see for example [13], [14]. In most cases, variations in the network topology are due to communication failures between two or more agents, which can be regarded as instantaneous with respect to the agents' dynamics. A large number of existing papers investigate pinning control for time-varying networks - see for example [15]-[18].

Pinning control algorithms have been traditionally designed in a continuous-time fashion. However, continuoustime distributed control laws are typically cumbersome or even not possible to be implemented for large-scale networks, mainly because in several scenarios the systems are supposed to communicate over a wireless medium, which represents a shared resource with limited capacity. In general, time-triggered control is not a viable solution to this issue, since it would require synchronization of the sampling in stants among all the agents and also their simultaneous transmission of information over the communication medium. Conversely, since the appearance of pioneering studies on the subject - such as [19], [20] - event-triggered approaches have been successfully developed for network synchronization problems - see [21]-[25] among the others.

The main contribution of this paper is to address the problem of multi-agent coordination with a network model that includes at the same time nonlinear dynamics of the individual agents, pinning control, event-triggered signals and time-varying topologies. Considering both static and timevarying networks of identical nonlinear dynamical systems, we design a model-based, distributed and event-triggered pinning control law which drives the states of such systems onto an a-priori specified common reference trajectory. We derive a set of sufficient conditions under which accumulation points in the sequences of events are excluded - which also implies absence of Zeno behavior [26] - and the agents achieve exponential convergence to the reference trajectory.

\section{Preliminaries}

For $x \in \mathbb{R}^{n}$ we denote $x_{[N]}=\left[x^{\top}, \ldots, x^{\top}\right]^{\top} \in \mathbb{R}^{n N} . I_{n}$ denotes the $n$-by- $n$ identity matrix.

Definition 2.1: A function $f: x \in \mathbb{R}^{n} \rightarrow \mathbb{R}^{n}$ is said to be globally Lipschitz with Lipschitz Constant $L_{f}$ if $\forall x, y \in$ $\mathbb{R}^{n}, \quad\|f(x)-f(y)\| \leq L_{f}\|x-y\|$.

Operator $\otimes$ between two matrices denotes their Kronecker product. On this operator, and also for the following lemma, see for example [27]. 
Lemma 2.1: For any two square matrices $A \in$ $\mathbb{R}^{n_{a} \times n_{a}}$ with eigenvalues $\lambda_{a}^{(1)}, \ldots, \lambda_{a}^{\left(n_{a}\right)}$ and eigenvectors $v_{a}^{(1)}, \ldots, v_{a}^{\left(n_{a}\right)}$, and $B \in \mathbb{R}^{n_{b} \times n_{b}}$ with eigenvalues $\lambda_{b}^{(1)}, \ldots, \lambda_{b}^{\left(n_{b}\right)}$ and eigenvectors $v_{b}^{(1)}, \ldots, v_{b}^{\left(n_{b}\right)}$ the eigenvalues of $A \otimes B$ are given by $\lambda_{a}^{(i)} \lambda_{b}^{(j)}$ and its eigenvectors are given by $v_{a}^{(i)} \otimes v_{b}^{(j)}$ with $i=1, \ldots, n_{a}$ and $j=1, \ldots, n_{b}$.

A graph $\mathcal{G}=\{\mathcal{V}, \mathcal{E}\}$ is defined by a set $\mathcal{V}=\{1, \ldots, N\}$ of nodes and a set $\mathcal{E} \subseteq \mathcal{V} \times \mathcal{V}$ of edges. A graph is called undirected if $(i, j) \in \mathcal{E} \Longleftrightarrow(j, i) \in \mathcal{E}$.

In an undirected graph two nodes $i$ and $j$ are said to be neighbors or adjacent if $(i, j) \in \mathcal{E}$. The number $d_{i}$ of neighbors of node $i$ is also called the degree of node $i$. A path from node $i$ to node $j$ is a sequence of nodes starting in $i$ and ending in $j$ such that every two consecutive nodes are adjacent. A graph is said to be connected if there exists a path between any two of its nodes. If a graph is not connected then the node set $\mathcal{V}$ can be partitioned into subsets $\mathcal{V}_{i}$ such that, denoting by $\mathcal{E}_{i}$ the restriction of $\mathcal{E}$ to $\mathcal{V}_{i}$, each of the graphs $\mathcal{G}_{i}:=\left\{\mathcal{V}_{i}, \mathcal{E}_{i}\right\}$ is connected. Such subsets are called the components of the original graph. The matrix

$$
\{A\}_{i j}= \begin{cases}1 & \text { if }(i, j) \in \mathcal{E} \\ 0 & \text { otherwise }\end{cases}
$$

is called adjacency matrix of the graph. Furthermore, the matrix

$$
\{L\}_{i j}= \begin{cases}\sum_{k=1}^{N} a_{i k} & \text { if } i=j \\ -a_{i j} & \text { if } i \neq j\end{cases}
$$

is called the Laplacian matrix of the graph. The Laplacian of any given undirected graph is symmetric with zero line sum, and therefore the vector $1_{[N]}$ is an eigenvector with null eigenvalue. It is also a known property of graph theory - see for example [1] - that the Laplacian is positive semidefinite, and it has as many null eigenvalues as many components there are in the graph.

Following [6], we associate a pin set $\mathcal{P} \subseteq \mathcal{V}$ to graph $\mathcal{G}$. If a node belongs to the pin set we say that it is pinned, or a pin. We say that the graph is pinned itself if every component contains at least one pinned node. We define

$$
\{P\}_{i j}= \begin{cases}1 & \text { if } i=j \text { and } i \in \mathcal{P} \\ 0 & \text { otherwise }\end{cases}
$$

as the pinning matrix of the graph. For any two positive scalars $c, \rho$ we say that the matrix

$$
L_{a}=c L+\rho P
$$

is an augmented Laplacian of the graph. Since it is a sum of two positive semidefinite matrices, an augmented Laplacian is itself positive semidefinite. We call its smallest eigenvalue $\lambda \geq 0$ augmented connectivity of the graph.

Lemma 2.2: The augmented connectivity of a graph is strictly positive if and only if the graph is pinned.

\section{Problem Statement}

We consider a network of $N$ interconnected agents with identical dynamics described by

$$
\dot{x}_{i}=f\left(x_{i}\right)+u_{i}, \quad x_{i} \in \mathbb{R}^{n} \quad \text { for } \quad i=1, \ldots, N .
$$

It is desired that the agents converge to a reference trajectory $s$ such that

$$
\dot{s}=f(s), \quad s \in \mathbb{R}^{n} .
$$

We introduce the tracking errors $e_{i}=s-x_{i}$ and the mismatches $e_{i j}=x_{j}-x_{i}=e_{i}-e_{j}$. We also introduce the stack vectors $x=\left[x_{1}^{\top}, \ldots, x_{N}^{\top}\right]^{\top}, e=$ $\left[e_{1}^{\top}, \ldots, e_{N}^{\top}\right]^{\top}, u=\left[u_{1}^{\top}, \ldots, u_{N}^{\top}\right]^{\top}$ and $s_{[N]}=\left[s^{\top}, \ldots, s^{\top}\right]$ all belonging to $\mathbb{R}^{N n}$. Moreover, we denote $F(x)=$ $\left[f\left(x_{1}\right)^{\top}, \ldots, f\left(x_{N}\right)^{\top}\right]^{\top} \in \mathbb{R}^{N n}$. For convenience we denote $\eta=\|e\|$. The control objective is to achieve convergence of the agents' states to the reference, in the sense that

$$
\lim _{t \rightarrow+\infty} \eta(t)=0
$$

We describe the topology of the connections in the network by a graph $\mathcal{G}$, where each node represents an agent, each edge $(i, j)$ represents a feedback from agent $j$ to agent $i$ and the pin set represents the agents that directly receive a feedback from the reference. Similarly to what done in [25], we adopt the following event-triggered control law:

$$
u_{i}(t)=c \sum_{j=1}^{N} a_{i j} e_{i j}\left(t_{k_{i}}^{(i)}\right)+\rho p_{i i} e_{i}\left(t_{k_{i}}^{(i)}\right), t \in\left[t_{k_{i}}^{(i)}, t_{k_{i}+1}^{(i)}\right),
$$

where $a_{i j}$ and $p_{i i}$ are elements of the incidence and the pinning matrix respectively and $c, \rho$ are two positive scalars. Note that the control input $u_{i}$ remains constant in the interval $\left[t_{k_{i}}^{(i)}, t_{k_{i}+1}^{(i)}\right)$. Time instants $t_{k_{i}}^{(i)}$ in which signal $u_{i}$ is updated are called events related to agent $i$. For convenience we introduce the signals

$$
\begin{gathered}
\tilde{e}_{i j}(t)=e_{i j}\left(t_{k_{i}}^{(i)}\right)-e_{i j}(t) \text { for } t \in\left[t_{k_{i}}^{(i)}, t_{k_{i}+1}^{(i)}\right), \\
\tilde{e}_{i}(t)=e_{i}\left(t_{k_{i}}^{(i)}\right)-e_{i}(t) \text { for } t \in\left[t_{k_{i}}^{(i)}, t_{k_{i}+1}^{(i)}\right),
\end{gathered}
$$

so that at all times the control signals can be written as

$$
u_{i}=c \sum_{j=1}^{N} a_{i j}\left(e_{i j}+\tilde{e}_{i j}\right)+\rho p_{i i}\left(e_{i}+\tilde{e}_{i}\right) .
$$

In order to determine sequences $\left\{t_{k_{i}}^{(i)}\right\}_{k_{i}=0}^{+\infty}$ we introduce the threshold function:

$$
\varsigma(t)=\varsigma_{0} e^{-\lambda_{\varsigma} t}
$$

Sequence $\left\{t_{k_{i}}^{(i)}\right\}_{k_{i}=0}^{+\infty}$ is defined recursively as follows:

$$
\begin{aligned}
t_{k_{i}+1}^{(i)}= & \min \left\{t>t_{k_{i}}^{(i)}:\right. \\
& a_{i j}\left\|\tilde{e}_{i j}(t)\right\| \geq \varsigma(t) \text { for some } j \in \mathcal{V} \text { or } \\
& \left.p_{i i}\left\|\tilde{e}_{i}(t)\right\| \geq \varsigma(t)\right\} .
\end{aligned}
$$

Therefore, an event for agent $i$ occurs anytime $a_{i j}\left\|\tilde{e}_{i j}(t)\right\|$ or $p_{i i}\left\|\tilde{e}_{i}(t)\right\|$ exceeds the current value of the threshold $\varsigma(t)$. 
Assumption 3.1: Function $f$ is globally Lipschitz with Lipschitz constant $L_{f}$.

Assumption 3.2: Denoting $\alpha=\lambda-L_{f}$, it holds that $0<$ $\lambda_{\varsigma}<\alpha$.

Remark 3.1: In order to satisfy Assumption 3.2, it is necessary, but not sufficient, that the graph underlying the network is pinned according to Lemma 2.2. However, if the graph is pinned, Assumption 3.2 can always be satisfied using sufficiently large $c$ and $\rho$.

\section{Convergence on Static Topologies}

In this section we prove that the proposed algorithm achieves convergence of the agents' states to the reference trajectory. In order to do this, first we show that there is a lower bound on the inter-event time $t_{k_{i}+1}^{(i)}-t_{k_{i}}^{(i)}$ for all the event sequences, then we prove that the norm of the error stack $\eta(t)$ converges exponentially to zero.

Theorem 4.1: Let us consider a network (1) controlled with law (2)-(4). Then under Assumptions 3.1 and 3.2 there exists a positive inter-event time for all the sequences $\left\{t_{k_{i}}^{(i)}\right\}_{k_{i}=0}^{+\infty}$ and the norm of the error stack $\eta(t)$ converges exponentially to zero.

Proof: Let us consider the closed-loop dynamics of the error signals:

$$
\begin{aligned}
\dot{e}_{i} & =\dot{s}-\dot{x}_{i}=f(s)-f\left(x_{i}\right)-u_{i} \\
& =f(s)-f\left(x_{i}\right)-c \sum_{j=1}^{N} a_{i j}\left(e_{i j}+\tilde{e}_{i j}\right)-\rho p_{i i}\left(e_{i}+\tilde{e}_{i}\right) .
\end{aligned}
$$

If we denote with $l_{i}^{\top}$ and $p_{i}^{\top}$ the $i$-th row of the Laplacian and the pinning matrix respectively, we can rewrite the last expression as

$$
\begin{aligned}
\dot{e}_{i}= & f(s)-f\left(x_{i}\right)-\left[\left(c l_{i}^{\top}+\rho p_{i}^{\top}\right) \otimes I_{n}\right] e \\
& -c \sum_{j=1}^{N} a_{i j} \tilde{e}_{i j}-\rho p_{i i} \tilde{e}_{i} .
\end{aligned}
$$

If we denote the last two addends with $\xi_{i}$ we can rewrite

$$
\dot{e}_{i}=f(s)-f\left(x_{i}\right)-\left[\left(c l_{i}^{\top}+\rho p_{i}^{\top}\right) \otimes I_{n}\right] e-\xi_{i} .
$$

Denoting $\xi=\left[\xi_{1}^{\top}, \ldots, \xi_{N}^{\top}\right]^{\top}$, we can group the previous equations for $i=1, \ldots, N$ in

$$
\dot{e}=F\left(s_{[N]}\right)-F(x)-\left(L_{a} \otimes I_{n}\right) e-\xi .
$$

where $L_{a}=c L+\rho P$ is an augmented Laplacian of the graph. Note that thanks to the triggering condition we have $\left\|\tilde{e}_{i j}\right\|,\left\|\tilde{e}_{i}\right\|<\varsigma$, therefore $\left\|\xi_{i}\right\| \leq\left(c d_{i}+\rho p_{i i}\right) \varsigma$ and consequently $\|\xi\| \leq \Delta \varsigma$, where $\Delta$ is a positive constant whose expression depends on the augmented Laplacian. Now we can calculate

$$
e^{\top} \dot{e}=e^{\top}\left[F\left(s_{[N]}\right)-F(x)\right]-e^{\top}\left(L_{a} \otimes I_{n}\right) e-e^{\top} \xi .
$$

Accounting for Assumption 3.1, Lemma 2.1, and the upper bound on $\|\xi\|$, we can bound the previous expression with

$$
e^{\top} \dot{e} \leq L_{f} \eta^{2}-\lambda \eta^{2}+\eta \Delta \varsigma
$$

Now we can observe that

$$
\dot{\eta}=\frac{d}{d t}\|e\|=\frac{e^{\top} \dot{e}}{\|e\|} \leq\left(L_{f}-\lambda\right) \eta+\Delta \varsigma .
$$

Under Assumption 3.2 we have $\alpha=\lambda-L_{f}>0$, so we can write $\dot{\eta} \leq-\alpha \eta+\Delta \varsigma$, which can be integrated in $[0, t]$ as follows:

$$
\eta(t) \leq e^{-\alpha t} \eta(0)+\Delta \int_{0}^{t} e^{-\alpha(t-\tau)} \varsigma(\tau) d \tau .
$$

Substituting $\varsigma(\tau)$ with its expression it is possible to solve the integral and obtain

$$
\eta(t) \leq e^{-\alpha t} \eta(0)+\frac{\Delta \varsigma_{0}}{\alpha-\lambda_{\varsigma}}\left(e^{-\lambda_{\varsigma} t}-e^{-\alpha t}\right) .
$$

Remembering that by Assumption 3.2 we have $0<\lambda_{\varsigma}<\alpha$ we can rewrite

$$
\eta(t) \leq\left(\eta(0)+\frac{\Delta \varsigma_{0}}{\alpha-\lambda_{\varsigma}}\right) e^{-\lambda_{\varsigma} t}=k_{\eta} \varsigma(t) .
$$

where $k_{\eta}$ is a positive constant. Now we can observe that

$$
\tilde{e}_{i}=-\int_{t_{k_{i}}^{(i)}}^{t} \dot{e}_{i}(\sigma) d \sigma \Longrightarrow\left\|\tilde{e}_{i}\right\| \leq \int_{t_{k_{i}}^{(i)}}^{t}\left\|\dot{e}_{i}(\sigma)\right\| d \sigma .
$$

Accounting for (5) we can easily write

$$
\begin{aligned}
\left\|\dot{e}_{i}\right\| & \leq L_{f}\left\|e_{i}\right\|+\left(c \mid\left\|l_{i}^{\top}\right\|+\rho\left\|p_{i}^{\top}\right\|\right)\|e\|+\left\|\xi_{i}\right\| \\
& \leq L_{f}\|e\|+\left(c \sqrt{2} d_{i}+\rho p_{i i}\right)\|e\|+\left(c d_{i}+\rho p_{i i}\right) \varsigma .
\end{aligned}
$$

From (6) this implies also

$$
\left\|\dot{e}_{i}\right\| \leq \omega_{i} \varsigma
$$

where $\omega_{i}$ is a positive constant of expression $\omega_{i}=\left(L_{f}+\right.$ $\left.c \sqrt{2} d_{i}+\rho p_{i i}\right) k_{\eta}+c d_{i}+\rho p_{i i}$. Using (7) and remembering that $\varsigma(t)$ is nonincreasing we can also write

$$
\left\|\tilde{e}_{i}\right\| \leq \omega_{i} \int_{t_{k_{i}}^{(i)}}^{t} \varsigma(\tau) d \tau \leq \omega_{i} \varsigma\left(t_{k_{i}}^{(i)}\right)\left(t-t_{k_{i}}^{(i)}\right) .
$$

This means that in order to have $\left\|\tilde{e}_{i}\right\| \geq \varsigma$ we need that

$$
\omega_{i} \varsigma\left(t_{k_{i}}^{(i)}\right)\left(t-t_{k_{i}}^{(i)}\right) \geq \varsigma(t)=\varsigma\left(t_{k_{i}}^{(i)}\right) e^{-\lambda_{\varsigma}\left(t-t_{k_{i}}^{(i)}\right)},
$$

that is,

$$
\omega_{i}\left(t-t_{k_{i}}^{(i)}\right) \geq e^{-\lambda_{\varsigma}\left(t-t_{k_{i}}^{(i)}\right)}
$$

The above inequality has a lower-bounded solution, therefore we have proven that there exists a lower bound on the time needed to have $\left\|\tilde{e}_{i}\right\| \geq \varsigma$ after $t_{k_{i}}^{(i)}$. In the same way it is possible to prove that there exists a lower bound on the time needed to have $\left\|\tilde{e}_{i j}\right\| \geq \varsigma$ after $t_{k_{i}}^{(i)}$, but considering

$$
\tilde{e}_{i j}=-\int_{t_{k_{i}}^{(i)}}^{t} \dot{e}_{i j}(\sigma) d \sigma=\int_{t_{k_{i}}^{(i)}}^{t} \dot{e}_{i}(\sigma)-\dot{e}_{j}(\sigma) d \sigma
$$

and

$$
\left\|\tilde{e}_{i j}\right\| \leq \int_{t_{k_{i}}^{(i)}}^{t}\left\|\dot{e}_{i}(\sigma)\right\|+\left\|\dot{e}_{j}(\sigma)\right\| d \sigma
$$


If we now look at (6), we can conclude that the error stack norm $\eta(t)$ converges exponentially to zero.

Remark 4.1: Note that the existence of a constant lowerbound for the inter-event times $t_{k_{i}+1}^{(i)}-t_{k_{i}}^{(i)}$ is a stronger property than absence of Zeno behavior [26].

\section{Convergence on Switching Topologies}

This section extends the presented results to time-varying topologies. In particular, consider here a special class of time-varying topologies, the switching graphs.

Definition 5.1: A graph is said to be switching with a dwell time $\tau_{d}>0$ if

- the node set is constant;

- the edge set and the pin set may be modified at discrete time instants, but two consecutive variations regarding the same edge or the same pin node are separated at least by a time interval $\tau_{d}$.

Variations in the edge set and the pin set are called switchings.

Assumption 5.1: The underlying graph of network (1) is switching with dwell time $\tau_{d}>0$.

When the network is built on a switching graph, sequences $t_{k_{i}}^{(i)}$ must be defined differently in order to take into account the possible changes in the topology of interactions and control. Specifically, the control signal $u_{i}$ must be updated every time a switching involving the $i$-th agent occurs. Therefore we define sequence $\left\{t_{k_{i}}^{(i)}\right\}_{k_{i}=0}^{+\infty}$ as follows:

$$
\begin{aligned}
t_{k_{i}+1}^{(i)}= & \min \left\{t>t_{k_{i}}^{(i)}:\right. \\
& a_{i j}(t)\left\|\tilde{e}_{i j}(t)\right\| \geq \varsigma(t) \text { for some } j \in \mathcal{V} \text { or } \\
& a_{i j}(t) \neq a_{i j}\left(t_{k_{i}}^{(i)}\right) \text { for some } j \in \mathcal{V} \text { or } \\
& p_{i i}(t)\left\|\tilde{e}_{i}(t)\right\| \geq \varsigma \text { or } \\
& \left.p_{i i}(t) \neq p_{i i}\left(t_{k_{i}}^{(i)}\right)\right\},
\end{aligned}
$$

and the control law is given by (2)-(3), (8).

Assumption 5.2: There exist two positive constants $T, \psi$ such that, denoting $\alpha=c \lambda-L_{f}$, for any time instant $t \geq 0$ it holds that

$$
0<\lambda_{\varsigma}<\psi \leq \frac{1}{T} \int_{t}^{t+T} \alpha(\tau) d \tau
$$

Note that in order to satisfy Assumption 5.2 it is not necessary that the underlying graph is always pinned. However, it has to be pinned sufficiently often for (9) to hold in every interval $[t, t+T]$.

Theorem 5.1: Let us consider a network (1) controlled with law (2)-(3), (8). Then under assumptions 3.1, 5.1 and 5.2 sequences $\left\{t_{k_{i}}^{(i)}\right\}_{k_{i}=0}^{+\infty}$ do not present accumulation points and the norm of the error stack $\eta(t)$ converges exponentially to zero.

Proof: Assumption 5.1 excludes that sequences $\left\{t_{k_{i}}^{(i)}\right\}_{k_{i}=0}^{+\infty}$ present accumulation points of events generated by switchings. Still we have to prove that there are no accumulation points of events generated by conditions $a_{i j}(t)\left\|\tilde{e}_{i j}(t)\right\| \geq \varsigma(t)$ or $p_{i i}(t)\left\|\tilde{e}_{i}(t)\right\| \geq \varsigma(t)$. Reasoning as in the static case, we can still write $\dot{\eta} \leq-\alpha(t) \eta+\Delta(t) \varsigma$, with now $\alpha$ and $\Delta$ being time-varying. Since the network has only a finite number of possible configurations, there exist upper and lower bounds for both $\alpha$ and $\Delta$. Integration of the previous inequality over a time interval $[t, t+T]$ yields

$$
\begin{aligned}
\eta(t+T) \leq & e^{-\int_{t}^{t+T} \alpha(\tau) d \tau} \eta(t) \\
& +\Delta_{M} \int_{t}^{t+T} e^{-\int_{\tau}^{t+T} \alpha(\sigma) d \sigma} \varsigma(\tau) d \tau
\end{aligned}
$$

where $\Delta_{M}$ is an upper bound for $\Delta$. Under Assumption 5.2 we can write

$$
\begin{aligned}
\int_{\tau}^{t+T} \alpha(\sigma) d \sigma & =\int_{t}^{t+T} \alpha(\sigma) d \sigma-\int_{t}^{\tau} \alpha(\sigma) d \sigma \\
& \geq \psi T-\alpha_{M}(\tau-t)
\end{aligned}
$$

where $\alpha_{M}$ is an upper bound for $\alpha$. Therefore we can bound $\eta(t+T)$ with

$$
\begin{aligned}
\eta(t+T) \leq & e^{-\psi T} \eta(t) \\
& +\Delta_{M} e^{-\psi T} \int_{t}^{t+T} e^{\alpha_{M}(\tau-t)} \varsigma(\tau) d \tau .
\end{aligned}
$$

Substituting $\varsigma(\tau)$ with its expression (3) we obtain

$$
\begin{aligned}
\eta(t+T) \leq & e^{-\psi T} \eta(t) \\
& +\frac{\Delta_{M} e^{-\psi T}\left(e^{\left(\alpha_{M}-\lambda_{\varsigma}\right) T}-1\right)}{\alpha_{M}-\lambda_{\varsigma}} \varsigma(t) .
\end{aligned}
$$

For $t=k T$ the previous expression has the form of the dynamics of a scalar discrete-time system that we rewrite as

$$
\eta((k+1) T) \leq a \eta(k T)+b \varsigma(k T),
$$

with $a$ and $b$ positive constants. The above expression can be explicitly written as

$$
\eta(k T) \leq a^{k} \eta(0)+b \sum_{h=0}^{k-1} \varsigma(h T) a^{k-1-h} .
$$

Substituting the expressions of $a$ and $\varsigma(k T)$ we obtain

$$
\begin{aligned}
\eta(k T) & \leq e^{-\psi k T} \eta(0)+b e^{-\psi(k-1) T} \sum_{h=0}^{k-1} e^{\left(\psi-\lambda_{\varsigma}\right) h T} \\
& \leq e^{-\psi k T} \eta(0)+b e^{-\psi(k-1) T} \frac{e^{\left(\psi-\lambda_{\varsigma}\right) k T}-1}{e^{\left(\psi-\lambda_{\varsigma}\right) T}-1} \\
& \leq e^{-\psi k T} \eta(0)+b \frac{e^{\psi T}}{e^{\left(\psi-\lambda_{\varsigma}\right) T}-1} e^{-\lambda_{\varsigma} k T} .
\end{aligned}
$$

Taking into account that $\lambda_{\varsigma}<\psi$ we finally obtain

$$
\eta(k T) \leq\left(\eta(0)+b \frac{e^{\psi T}}{e^{\left(\psi-\lambda_{\varsigma}\right) T}-1}\right) e^{-\lambda_{\varsigma} k T}=k_{\eta}^{\prime} \varsigma(k T),
$$

where $k_{\eta}^{\prime}$ is a positive constant. Observing that $\alpha=\lambda-L_{f}$ is lower bounded by $-L_{f}$, we can write

$$
\dot{\eta} \leq L_{f} \eta+\Delta_{M \varsigma}
$$


which integrated over an interval $[k T, t]$ with $k T \leq t<$ $(k+1) T$ becomes

$$
\begin{aligned}
\eta(t) \leq & e^{L_{f}(t-k T)} \eta(k T)+\Delta_{M} \varsigma_{0} \int_{k T}^{t} e^{L_{f}(t-\tau)} e^{-\lambda_{\varsigma} \tau} d \tau \\
\leq & e^{L_{f}(t-k T)} \eta(k T) \\
& +\Delta_{M} \varsigma_{0} e^{L_{f} t} \frac{e^{-\left(L_{f}+\lambda_{\varsigma}\right) k T}-e^{-\left(L_{f}+\lambda_{\varsigma}\right) t}}{L_{f}+\lambda_{\varsigma}} \\
\leq & e^{L_{f} T}\left(\eta(k T)+\frac{\Delta_{M}}{L_{f}+\lambda_{\varsigma}} \varsigma(k T)\right) .
\end{aligned}
$$

Taking into account (10), the previous inequality yields

$$
\eta(t) \leq k_{\eta}^{\prime \prime} \varsigma(k T),
$$

where $k_{\eta}^{\prime \prime} \varsigma(k T)$ is yet another known positive constant. Now since $k T \leq t<(k+1) T$ we have $\varsigma(k T)=e^{\lambda_{\varsigma} T} \varsigma((k+$ 1)T) $\leq e^{\lambda_{\varsigma} T} \varsigma(t)$, which leads to

$$
\eta(t) \leq k_{\eta}^{\prime \prime} e^{\lambda_{\varsigma} T} \varsigma(t)
$$

Since such reasoning is valid for all $k=0,1, \ldots$, inequality (11) is valid at all times $t \geq 0$. Now, focusing on $\left\|\dot{e}_{i}\right\|$ and reasoning as we did in the static case, we can write

$$
\left\|\dot{e}_{i}\right\| \leq L_{f}\|e\|+(c \sqrt{2}(N-1)+\rho)\|e\|+(c N+\rho) \varsigma .
$$

Note that in this case, despite the fact that $d_{i}$ and $p_{i i}$ are timevarying, it holds that $d_{i} \leq N-1$ and $p_{i i} \leq 1$. Accounting for (11), inequality (12) implies that there exists a constant $\omega$ such that $\left\|\dot{e}_{i}\right\| \leq \omega \varsigma$. Reasoning exactly like in the static case we obtain a lower bound on the time needed to get $\left\|\tilde{e}_{i j}\right\| \geq \varsigma$ for some $j \in \mathcal{V}$ or $\left\|\tilde{e}_{i}\right\| \geq \varsigma$ after $t_{k_{i}}^{(i)}$.

If we now look at (11), we can conclude that the error stack norm $\eta(t)$ converges exponentially to zero.

Remark 5.1: Since events can be generated by switchings, which are exogenous with respect to the agents' dynamics, two consecutive updates of signal $u_{i}$, one caused by a switching and one caused by $\left\|\tilde{e}_{i}\right\|$ or some $\left\|\tilde{e}_{i j}\right\|$ meeting the threshold function, may be arbitrarily close in time. For this reason, although we proved that no accumulation points exist, in order to cope with switching topologies, our control algorithm has to rely on fast actuators. However, note that absence of accumulation points in sequences $\left\{t_{k_{i}}^{(i)}\right\}_{k_{i}=0}^{+\infty}$ is still a stronger property than absence of Zeno behavior [26].

\section{NUMERICAL EXAMPLES}

To illustrate the effectiveness of the proposed control algorithm, we apply it to a simulated network. We consider a set of $N=10$ identical Chua oscillators. The individual dynamics of each oscillator are described by

$$
f(x)=\left[\begin{array}{c}
a\left(x_{2}-x_{1}-\phi\left(x_{1}\right)\right) \\
x_{1}-x_{2}+x_{3} \\
-b x_{2}
\end{array}\right],
$$

where $\phi(y)=m_{1} y+\frac{1}{2}\left(m_{0}-m_{1}\right)(|y+1|-|y-1|) \forall y \in$ $\mathbb{R}$. Choosing $a=b=1, m_{0}=-1.5, m_{1}=-0.5$, the oscillators are globally Lipschitz with $L_{f} \simeq 3.75$ - see [25].

The oscillators are connected over a network with 25 edges - out of a maximum of 45 possible edges - a coupling gain

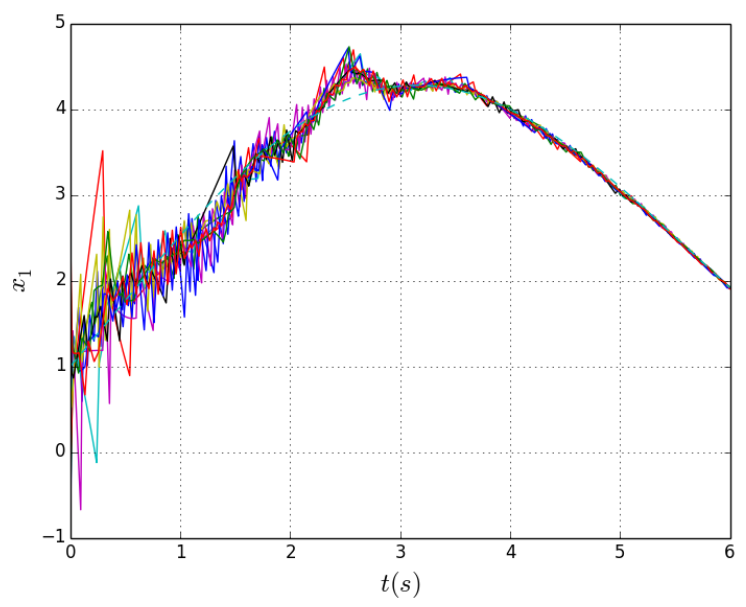

Fig. 1. Trend of the first state variable for all the agents (solid) and the reference (dashed) during the described simulation.

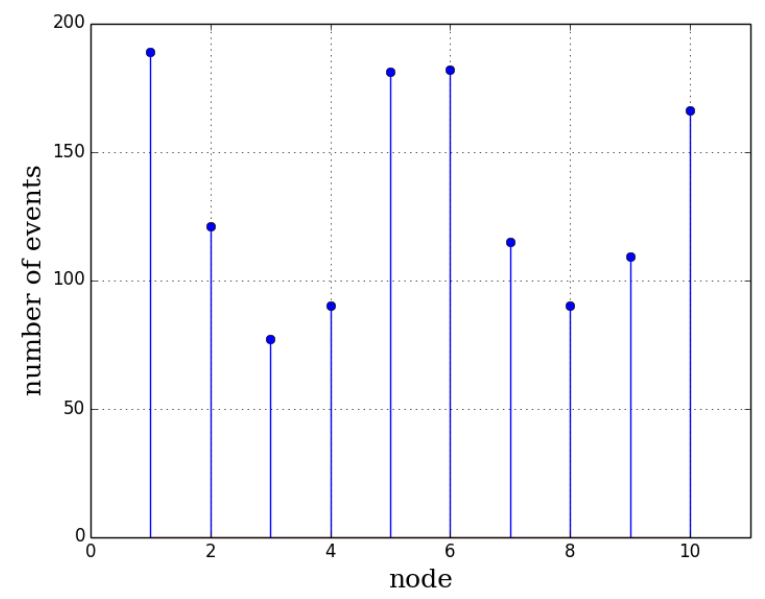

Fig. 2. Number of events occurred for each agent in the network during the described simulation.

$c=8$ and a control gain $\rho=25$. Our simulation is set on a time interval $[0,6] \mathrm{s}$. At the beginning of the experiment the three nodes with highest degrees are pinned, which yields $\lambda \simeq 5.29$. At $t=0.75 \mathrm{~s}$ one pin is removed, which causes $\lambda \simeq 3.36$. At $t=0.90 \mathrm{~s}$ the two remaining pins are removed as well, which causes $\lambda=0$. At $t=1.0 \mathrm{~s}$ the original pinning scheme is restored and the cycle repeats itself every second. It is easy to see that Assumption 5.1 holds with $\tau_{d}=1 \mathrm{~s}$. If we set $T=1 s$, we can calculate

$$
\begin{aligned}
\psi & =\frac{1}{T} \int_{t}^{T} \alpha(\tau) d \tau \\
& \simeq 5.29 \cdot .75+3.36 \cdot .15-3.75 \simeq .72 .
\end{aligned}
$$

For the threshold function, we pick $\varsigma_{0}=3.0$ and $\lambda_{\varsigma}=$ 0.70 , so that Assumption 5.2 holds. For all the oscillators, the initial state values are chosen in the interval $[-1.0,1.0]$. The simulation is run with with a time step of $1.0 \mathrm{~ms}$. 


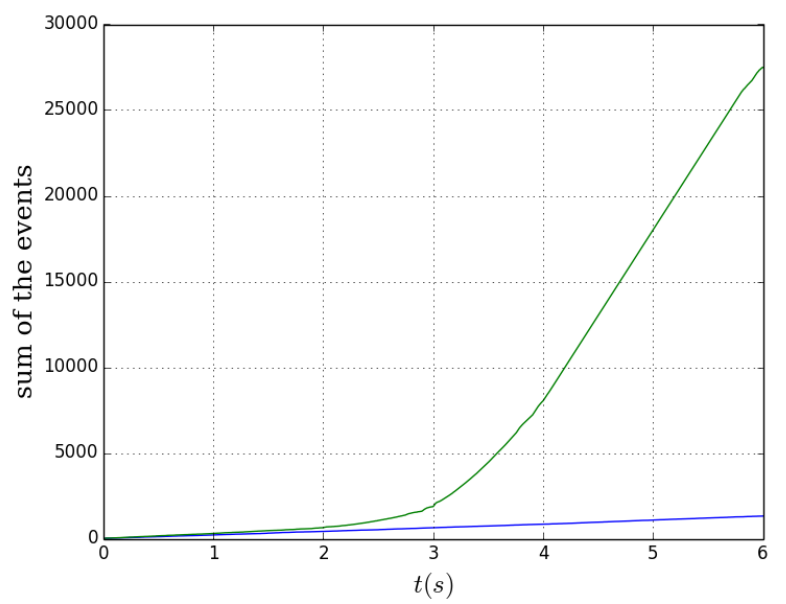

Fig. 3. Trend of the sum of the events occurred in the network during the simulation with $\lambda_{\varsigma}=0.70$ (blue) and during the simulation with $\lambda_{\varsigma}=7.0$ (green).

Figure 1 shows the trend of the first state variable of all the agents and the reference, during the experiment while Figure 2 shows the total number of events for each agent. It is possible to observe that all the agents update their control signal less than 200 times during the experiment, so less than 34 times per second on average.

In order to show the importance of selecting an appropriate threshold function, we repeat the same simulation using $\lambda_{\varsigma}=$ 7.0. Note that with this choice of parameters Assumption 5.2 is not met. Figure 3 shows the trend of the sum of the events occurred for all the agents in the network, both for the case $\lambda=0.70$ (blue curve) and for the case $\lambda=7.0$ (green curve). For the first case it is possible to see how the total number of events grows linearly with respect to time with a constant slope, thus indicating that, as expected, we do not have an accumulation of events for any $t$. Conversely, in the second case the total amount of events sensibly deviates from the previous curve. The increase of slope for increasing $t$ indicates a continuous increment in the number of events. This is consistent with the fact that, violating Assumption 5.2, the threshold (3) is shrinking faster than the network dynamics.

\section{CONCLUSIONS}

In this paper we presented a novel model-based distributed algorithm for event-triggered pinning control of a network of nonlinear dynamical systems. The concepts of pinned graph and augmented connectivity have been introduced. Convergence of the proposed algorithm has been proven under opportune hypotheses for both static and switching networks. A numerical example has been presented to validate the theoretical results.

\section{REFERENCES}

[1] R. Olfati-Saber, J. A. Fax, and R. M. Murray. Consensus and cooperation in networked multi-agent systems. Proceedings of the IEEE, 95(1):215-233, 2007.
[2] A. Arenas, A. Díaz-Guilera, J. Kurths, Y. Moreno, and C. Zhou. Synchronization in complex networks. Physics Reports, 469(3):93$153,2008$.

[3] X. F. Wang and G. Chen. Pinning control of scale-free dynamical networks. Physica A: Statistical Mechanics and its Applications, 310(3):521-531, 2002.

[4] L. Y. Xiang, Z. X. Liu, Z. Q. Chen, F. Chen, and Z. Z. Yuan. Pinning control of complex dynamical networks with general topology. Physica A: Statistical Mechanics and its Applications, 379(1):298306, 2007.

[5] F. Sorrentino, M. di Bernardo, F. Garofalo, and G. Chen. Controllability of complex networks via pinning. Physical Review E, 75(4), 2007.

[6] M. Porfiri and M. Di Bernardo. Criteria for global pinningcontrollability of complex networks. Automatica, 44(12):3100-3106, 2008.

[7] W. Wu, W. Zhou, and T. Chen. Cluster synchronization of linearly coupled complex networks under pinning control. Circuits and Systems I: Regular Papers, IEEE Transactions on, 56(4):829-839, 2009.

[8] Y. Y. Liu, J. J. Slotine, and A. L. Barabási. Controllability of complex networks. Nature, 473(7346):167-173, 2011.

[9] X. Li, X. Wang, and G. Chen. Pinning a complex dynamical network to its equilibrium. Circuits and Systems I: Regular Papers, IEEE Transactions on, 51(10):2074-2087, 2004.

[10] W. Lu, X. Li, and Z. Rong. Global stabilization of complex networks with digraph topologies via a local pinning algorithm. Automatica, 46(1):116-121, 2010.

[11] T. Chen, X. Liu, and W. Lu. Pinning complex networks by a single controller. Circuits and Systems I: Regular Papers, IEEE Transactions on, 54(6):1317-1326, 2007.

[12] J. Zhou, J. Lu, and J. Lü. Pinning adaptive synchronization of a general complex dynamical network. Automatica, 44(4):996-1003, 2008.

[13] I. V. Belykh, V. N. Belykh, and M. Hasler. Blinking model and synchronization in small-world networks with a time-varying coupling. Physica D: Nonlinear Phenomena, 195(1):188-206, 2004.

[14] L. Wang and Q. Wang. Synchronization in complex networks with switching topology. Physics Letters A, 375(34):3070-3074, 2011.

[15] W. Xia and J. Cao. Pinning synchronization of delayed dynamical networks via periodically intermittent control. Chaos: An Interdisciplinary Journal of Nonlinear Science, 19(1), 2009.

[16] G. Shi and K. H. Johansson. Multi-agent robust consensus-part i: Convergence analysis. In IEEE Conference on Decision and Control and European Control Conference, Orlando, FL, USA.

[17] G. Shi and K. H. Johansson. Multi-agent robust consensus-part ii: Application to distributed event-triggered coordination. In IEEE Conference on Decision and Control and European Control Conference, Orlando, FL, USA, 2011.

[18] M. Frasca, A. Buscarino, A. Rizzo, and L. Fortuna. Spatial pinning control. Physical review letters, 108(20), 2012.

[19] P. Tabuada. Event-triggered real-time scheduling of stabilizing control tasks. Automatic Control, IEEE Transactions on, 52(9):1680-1685, 2007.

[20] W. P. M. H. Heemels, J. H. Sandee, and P. P. J. Van Den Bosch. Analysis of event-driven controllers for linear systems. International journal of control, 81(4):571-590, 2008.

[21] D. V. Dimarogonas and K. H. Johansson. Event-triggered control for multi-agent systems. In IEEE Conference on Decision and Control and Chinese Control Conference, Shanghai, China, 2009.

[22] D. V. Dimarogonas, E. Frazzoli, and K. H. Johansson. Distributed event-triggered control for multi-agent systems. Automatic Control, IEEE Transactions on, 57(5):1291-1297, 2012.

[23] G. S. Seyboth, D. V. Dimarogonas, and K. H. Johansson. Eventbased broadcasting for multi-agent average consensus. Automatica, 49(1):245-252, 2013.

[24] W. P. M. H. Heemels, K. H. Johansson, and P. Tabuada. An introduction to event-triggered and self-triggered control. In Decision and Control, IEEE Conference on, Maui, Hawaii, US, 2012.

[25] D. Liuzza, D. V. Dimarogonas, M. di Bernardo, and K. H. Johansson. Distributed model-based event-triggered control for synchronization of multi-agent systems. In IFAC Conference on Nonlinear Control Systems, NOLCOS, Toulouse, France, 2013.

[26] K. H. Johansson, M. Egerstedt, J. Lygeros, and S. Sastry. On the regularization of zeno hybrid automata. Systems \& Control Letters, 38(3):141-150, 1999.

[27] R. A. Horn and C. R. Johnson. Topics in Matrix Analysis. Cambridge University Press, 1991. 